\title{
Tendencia de los años perdidos de vida potencial por trauma en Colombia: análisis de un periodo de nueve años
}

\author{
Trend of lost years of potential life due to trauma in Colombia: \\ Analysis of a nine-year period
}

\author{
Juan Sanjuán ${ }^{1}$, Rolando Medina², Wilmer Botache ${ }^{3}$, Francisco Montoya ${ }^{4}$, Gabriela Ruiz \\ Alberto F. García ${ }^{6}$, Marisol Badiel7 , Carlos A. Ordóñez ${ }^{8}$
}

1 Médico, cirujano general, M.Sc. en Epidemiología, Hospital Hernando Moncaleano Perdomo, Neiva, Colombia; profesor invitado, Universidad Surcolombiana, Neiva, Colombia; jefe, Grupo de Investigaciones CYTRA, Neiva, Colombia

2 Médico, cirujano de Trauma y Emergencias, especialista en Epidemiología, Hospital Hernando Moncaleano Perdomo de Neiva, Neiva, Colombia; coordinador, especialización en Cirugía General, Universidad Surcolombiana, Neiva, Colombia

3 Médico, cirujano de Trauma y Emergencias, Ph.D. en Bioética (candidato), Hospital Hernando Moncaleano Perdomo, Neiva, Colombia; docente, especialización en Cirugía General, Universidad Surcolombiana, Neiva, Colombia

4 Estudiante de Medicina, Universidad Surcolombiana, Neiva, Colombia

5 Estudiante de Medicina, Fundación Universitaria Juan N. Corpas, Bogotá, D.C., Colombia

6 Médico, cirujano de Trauma y Emergencias, M.Sc. en Epidemiología, Unidad de Cuidado Intensivo y Soporte Nutricional, Fundación Valle del Lili, Cali, Colombia; profesor, Universidad ICESI y Universidad del Valle, Cali, Colombia

7 Médica; decana, Facultad de Salud, Universidad Libre, Cali, Colombia

8 Médico, cirujano de Trauma y Emergencias y Unidad de Cuidado Intensivo, Fundación Valle del Lili, Cali, Colombia; Departamento de Cirugía General, Hospital Universitario del Valle, Cali, Colombia; profesor, Universidad ICESI y Universidad del Valle, Cali, Colombia

\section{Resumen}

Introducción. El trauma es reconocido como una epidemia global que varía según las regiones donde se presenta. Una parte de la carga de la enfermedad está determinada por los años perdidos de vida potencial, la cual es una estimación de amplio uso para la vigilancia en salud pública; sin embargo, existe poca información disponible en relación a esto. El objetivo de este estudio fue determinar la tendencia de los años perdidos de vida potencial por el trauma en un período de nueve años.

Métodos. La información se obtuvo del Instituto Nacional de Medicina Legal y Ciencias Forenses en un período de nueve años (2007-2015). La población de referencia se determinó con base en las proyecciones del Departamento Administrativo Nacional de Estadística, que indicaron una expectativa de vida de 75 años.

Resultados. En el 2015, a las lesiones por traumatismos en Colombia les correspondieron 1.920,7 años perdidos de vida potencial por cada Ioo.ooo personas. Durante el período de estudio, las principales causas fueron los

Fecha de recibido: 11/07/2018 - Fecha aceptación: 10/10/2018

Correspondencia: Juan Sanjuán, MD, Calle 21 sur \#22-63 Torre 4 Apartamento 301, Neiva, Colombia. Teléfono 316-8202016 Correo electrónico: drjuansanjuan@gmail.com

Citar como: Sanjuán J, Medina R, Botache W, Montoya F, Ruiz G, García AF, et al. Tendencia de los años perdidos de vida potencial por trauma en Colombia: análisis de un periodo de nueve años. Rev Colomb Cir. 2019;34:346-53. https://doi.org/10.30944/20117582.513.

Este es un artículo de acceso abierto bajo una Licencia Creative Commons - BY-NC-ND https://creativecommons.org/licenses/by-nc-nd/4.0/deed.es 
homicidios (rango, 5I a $68 \%$ ) y los accidentes de tránsito (rango, I9 a $28 \%$ ); la relación entre hombres y mujeres fue de 7:I, y la tendencia estadística fue hacia la disminución de los años perdidos de vida potencial.

Conclusiones. Los homicidios siguen aportando el mayor número de lesiones por trauma y años perdidos de vida potencial. Los hombres jóvenes continuaron siendo la población mayormente afectada. Se conceptúa la necesidad de incrementar los esfuerzos para mejorar la vigilancia en salud pública y ahondar en las intervenciones oportunas relacionadas con el trauma.

Palabras clave: años potenciales de vida perdidos; heridas y lesiones; homicidio; accidentes de tránsito; análisis socioeconómico; salud pública.

\begin{abstract}
Introduction: Trauma is a worldwide leading cause of external injuries that varies according to the regions. In 2015, trauma injuries were the third cause of Disability Adjusted Life Years (DALYs) with the $9 \%$ of the total global burden of disease. A portion of the burden of disease is determined by the Years of Potential Life Lost (YPLL). In Colombia in 2015, from the total of deaths due to external cause injuries, homicides had the highest number of YPLL with a total of 495.667; traffic accidents had 236.237 YPLL and accidental deaths 90.745 YPLL. The YPLL trauma trends and baselines are important to public health surveillance but there's no consolidated description. The aim of this study is to determine trauma trends in a five-year period.

Material and methods: The information was obtained from the reports of Instituto Nacional de Medicinal Legal y Ciencias Forenses in a nine-year period (2007-2015). The reference population was identified through the population projections from the Departmento Administrativo Nacional de Estadistica (DANE). YPLL calculation was compared to a 75 years life expectancy.

Results: In 2015 trauma injuries in Colombia had a total of I.920,7 YPLL per I00.00o people. Overall the study period, leading cause of YPLL was homicides (range $=5 \mathrm{I}-68 \%$ ) and traffic accidents (range $=19-28 \%$ ), ratio male: female was 7:I and YPLL observed had decreased. The percentage of the total decrease was $6.3 \%$, the highest increase was observed in 2009 with a raise of $30.5 \%$.

Conclusions: Homicides are a major public health issue such as the leading cause in YPLL of trauma injuries. Despite there was no increase in sex ratio, younger males are getting more affected trough the time increasing YPLL in this population group. More efforts are needed to improve public health surveillance for assessing baselines, DALYs, policies and evidence for interventions in trauma-related injuries.
\end{abstract}

Key words: potential years of life lost; wounds and injuries; homicide; accidents, traffic; socioeconomic analysis; public health.

\title{
Introducción
}

El trauma es reconocido como una epidemia global que varía según las regiones donde se presenta ${ }^{\mathrm{I}, 2}$. Es una causa importante de mortalidad y de discapacidad en el mundo, con aumento creciente en los últimos 20 años, y se relaciona con el nivel socioeconómico, el desempleo, las modificaciones en el tiempo y el desarrollo de cada región ${ }^{3-12}$. Una manera de medir el impacto del trauma es mediante el cálculo de los años perdidos de vida potencial respecto a la expectativa de vida de la sociedad en la que se presenta.

En Colombia, las muertes ocasionadas por traumas (homicidios, suicidios, muertes accidentales y accidentes de tránsito) son estudiadas por 
el Instituto de Medicina Legal y Ciencias Forenses, y publicadas en la revista Forensis, datos para la vida, en la cual se encuentran discriminadas por grupos de edad y mecanismo de las lesiones relacionadas. Según los resultados publicados para el año 20I5, el homicidio es la primera causa de muerte por causa externa, con la pérdida de 454.355 años de vida potencial, seguido por los accidentes de tránsito, con 217.763 años, y por las muertes por accidentes, con 83.734 años ${ }^{13}$.

Algunos de los resultados sobre el comportamiento de la mortalidad relacionada con el trauma para el periodo de 1985 a 2006 , describen la mortalidad por homicidios como la causa más importante de años perdidos de vida potencial en hombres y en mujeres ${ }^{14}$. Sin embargo, para analizar el impacto del trauma de manera general y como problema de salud pública enmarcado en diferentes eventos, es necesario conocer el impacto de todas las causas relacionadas, así como la tendencia en el tiempo del número de años perdidos de vida potencial en los últimos cinco años de los cuales se disponga información.

El objetivo de este trabajo fue determinar los años perdidos de vida potencial por traumas (homicidios, accidentes de tránsito y muertes accidentales) y conocer su tendencia en el periodo de 2007 a 2015 en Colombia.

\section{Métodos}

Se llevó a cabo un estudio observacional descriptivo. Los datos se obtuvieron de los reportes del Instituto Nacional de Medicina Legal y Ciencias Forenses publicados en Forensis, para el periodo comprendido entre el 2007 y el 2015. Para el cálculo de los años perdidos de vida potencial, se tuvo en cuenta la información por edad y sexo.

La captura de la información se hizo en Excel $^{\mathrm{TM}}$. Se excluyó la información reportada cuando la edad o el sexo no se habían anotado. Los denominadores de la población se identificaron según las proyecciones de la población del Departamento Administrativo Nacional de Estadística (DANE).
Los cálculos de los años perdidos de vida potencial se hicieron mediante un análisis de intervalos de clase y se utilizó una expectativa de vida de 75 años. Los datos se procesaron según las directrices de la Organización Panamericana de la Salud (OPS) ${ }^{15}$. Las variables categóricas se resumieron mediante frecuencias absolutas (n) y relativas (\%), y las continuas, mediante la mediana y el rango intercuartílico (RIC). No se plantearon comparaciones estadísticas. Los datos y las gráficas se procesaron en Excel ${ }^{\mathrm{TM}}$.

\section{Resultados}

La distribución de la edad, el sexo y la causa de muerte por año de reporte en el periodo del 2007 al 2015 se pueden apreciar en la tabla I.

En relación a la tendencia, el mayor número de años perdidos de vida potencial por Ioo.00o habitantes corresponde al año 20Io, en el cual se observaron de 2.576 años en total y la reducción para el año 20II fue de 4,5\% (II5 años perdidos de vida potencial por I0o.0oo habitantes) (tabla 2). Sin embargo, el mayor incremento se observó en el año 2009, con un aumento de 30,5 \% (600,I años perdidos de vida potencial por I00.00o habitantes) respecto al año 2008. Durante el periodo de nueve años, hubo una disminución del $6,3 \%$ (I30 años perdidos de vida potencial por Ioo.ooo habitantes).

Durante el año 2009, el aumento de años perdidos de vida potencial en los hombres fue de $29,8 \%$ (I.044,2 por Ioo.00o habitantes) y, en las mujeres, del 34,5 \% (I59,4 años perdidos de vida potencial por IOo.0oo habitantes) (figura I). Los años perdidos de vida potencial difirieron según el sexo, hubo un mayor número en hombres en el periodo de cinco años. El mayor número de años perdidos de vida potencial por I00.00o habitantes en los hombres, se presentó en el año 2009 y su reducción al 2015 fue del 26,0 \% (I.I88,4 años perdidos de vida potencial por Ioo.00o habitantes). En las mujeres, el mayor número de años perdidos de vida potencial por Ioo.0oo habitantes se encontró en el año 2010 y su reducción al 2015 fue de $23,5 \%$ (I54,I años perdidos de vida potencial por IOO.00o habitantes). 
Tabla 1. Causas de muerte por lesiones externas por año, según grupos de edad en el periodo de nueve años entre 2007 y 2015

\begin{tabular}{|c|c|c|c|c|c|c|c|c|c|}
\hline & $\begin{array}{l}2007 \\
\text { n (\%) }\end{array}$ & $\begin{array}{l}2008 \\
\text { n (\%) }\end{array}$ & $\begin{array}{l}2009 \\
\text { n (\%) }\end{array}$ & $\begin{array}{l}2010 \\
\text { n (\%) }\end{array}$ & $\begin{array}{l}2011 \\
\text { n (\%) }\end{array}$ & $\begin{array}{l}2012 \\
\text { n (\%) }\end{array}$ & $\begin{array}{l}2013 \\
\text { n (\%) }\end{array}$ & $\begin{array}{l}2014 \\
\text { n (\%) }\end{array}$ & $\begin{array}{l}2015 \\
\text { n (\%) }\end{array}$ \\
\hline & 21.424 & 20.685 & 27.169 & 27.451 & 26.568 & 25.716 & 23.964 & 22.548 & 22.124 \\
\hline Edad (años) ${ }^{*}$ & $31(24-42)$ & $31(23-42)$ & $30(23-42)$ & $25(23-42)$ & $30(23-43)$ & $30(29-43)$ & $30(29-43)$ & $31(28-44)$ & $31(29-44)$ \\
\hline Hombres & $\begin{array}{c}18.899 \\
(88,2)\end{array}$ & $\begin{array}{c}18.266 \\
(88,3)\end{array}$ & $\begin{array}{c}23.852 \\
(87,8)\end{array}$ & $\begin{array}{c}24.047 \\
(87,6)\end{array}$ & $\begin{array}{c}23.275 \\
(87,6)\end{array}$ & $\begin{array}{c}22.590 \\
(87,8)\end{array}$ & $\begin{array}{c}21.050 \\
(87,8)\end{array}$ & $\begin{array}{c}19.582 \\
(86,8)\end{array}$ & $\begin{array}{c}19.254 \\
(87)\end{array}$ \\
\hline \multicolumn{10}{|l|}{ Causas } \\
\hline Accidentales & $\begin{array}{c}393 \\
(1,8)\end{array}$ & $\begin{array}{c}212 \\
(1,0)\end{array}$ & $\begin{array}{c}2.590 \\
(9,5)\end{array}$ & $\begin{array}{l}3.034 \\
(11,1)\end{array}$ & $\begin{array}{l}2.955 \\
(11,1)\end{array}$ & $\begin{array}{l}2.614 \\
(10,2)\end{array}$ & $\begin{array}{c}2.303 \\
(9,6)\end{array}$ & $\begin{array}{l}2.387 \\
(10,5)\end{array}$ & $\begin{array}{l}2.342 \\
(10,6)\end{array}$ \\
\hline Homicidio & $\begin{array}{c}14.645 \\
(68,3)\end{array}$ & $\begin{array}{l}13.927 \\
(67,3)\end{array}$ & $\begin{array}{c}17.459 \\
(64,2)\end{array}$ & $\begin{array}{l}17.351 \\
(63,2)\end{array}$ & $\begin{array}{c}16.431 \\
(61,8)\end{array}$ & $\begin{array}{c}15.619 \\
(60,7)\end{array}$ & $\begin{array}{c}14.199 \\
(59,2)\end{array}$ & $\begin{array}{c}12.512 \\
(55,5)\end{array}$ & $\begin{array}{l}11.495 \\
(51,9)\end{array}$ \\
\hline Suicidio & $\begin{array}{l}1.552 \\
(7,2)\end{array}$ & $\begin{array}{c}1.619 \\
(7,8)\end{array}$ & $\begin{array}{c}1.784 \\
(6,6)\end{array}$ & $\begin{array}{l}1.799 \\
(6,6)\end{array}$ & $\begin{array}{c}1.829 \\
(6,9)\end{array}$ & $\begin{array}{c}1.823 \\
(7)\end{array}$ & $\begin{array}{c}1.751 \\
(7,3)\end{array}$ & $\begin{array}{c}1.807 \\
(8)\end{array}$ & $\begin{array}{c}1.976 \\
(8,9)\end{array}$ \\
\hline Tránsito & $\begin{array}{l}4.834 \\
(22,6)\end{array}$ & $\begin{array}{l}4.927 \\
(23,9)\end{array}$ & $\begin{array}{l}5.336 \\
(19,6)\end{array}$ & $\begin{array}{l}5.267 \\
(19,2)\end{array}$ & $\begin{array}{l}5.353 \\
(20,1)\end{array}$ & $\begin{array}{c}5.660 \\
(22)\end{array}$ & $\begin{array}{l}5.711 \\
(23,8)\end{array}$ & $\begin{array}{l}5.842 \\
(25,9)\end{array}$ & $\begin{array}{l}6.311 \\
(28,5)\end{array}$ \\
\hline
\end{tabular}

*Mediana (rango intercuartílico)

Tabla 2. Número de muertes y años perdidos de vida potencial distribuidos según grupos de edad en el periodo de nueve años entre 2007 y 2015.

\begin{tabular}{|c|c|c|c|c|c|c|c|c|c|}
\hline Edad (años) & 2007 & 2008 & 2009 & 2010 & 2011 & 2012 & 2013 & 2014 & 2015 \\
\hline 1 a 4 & 167 & 212 & 445 & 451 & 434 & 399 & 346 & 379 & 338 \\
\hline 5 a 9 & 147 & 131 & 233 & 283 & 268 & 233 & 206 & 218 & 194 \\
\hline 10 a 14 & 289 & 318 & 438 & 492 & 460 & 472 & 416 & 395 & 371 \\
\hline 15 a 19 & 2.101 & 2.090 & 2.853 & 3.038 & 3.117 & 3.033 & 2.879 & 2.742 & 2.601 \\
\hline 20 a 24 & 3.613 & 3.593 & 4.560 & 4.601 & 4.512 & 4.522 & 4.196 & 3.928 & 3.811 \\
\hline 25 a 29 & 3.831 & 3.500 & 4.714 & 4.505 & 4.207 & 3.938 & 3.651 & 3.194 & 3.220 \\
\hline 30 a 34 & 2.839 & 2.810 & 3.527 & 3.556 & 3.391 & 3.141 & 2.977 & 2.792 & 2.696 \\
\hline 35 a 39 & 2.334 & 2.197 & 2.633 & 2.614 & 2.485 & 2.317 & 2.142 & 1.984 & 2.025 \\
\hline 40 a 44 & 1.830 & 1.685 & 2.158 & 2.230 & 2.042 & 1.951 & 1.774 & 1.621 & 1.517 \\
\hline 45 a 49 & 1.253 & 1.284 & 1.779 & 1.707 & 1.645 & 1.649 & 1.480 & 1.412 & 1.337 \\
\hline 50 a 54 & 961 & 977 & 1.239 & 1.324 & 1.284 & 1.262 & 1.220 & 1.180 & 1.212 \\
\hline 55 a 59 & 638 & 652 & 936 & 951 & 986 & 1.034 & 945 & 902 & 983 \\
\hline 60 a 64 & 630 & 488 & 624 & 747 & 676 & 701 & 692 & 728 & 760 \\
\hline 65 a 69 & 438 & 394 & 561 & 509 & 560 & 528 & 573 & 554 & 569 \\
\hline 70 a 74 & 353 & 354 & 469 & 443 & 501 & 536 & 467 & 519 & 490 \\
\hline Total & 21.424 & 20.685 & 27.169 & 27.451 & 26.568 & 25.716 & 23.964 & 22.548 & 22.124 \\
\hline APVP & 879.500 & $854.362,5$ & $1 ' 127.857,5$ & $1 ' 142.042,5$ & 1'103.200 & 1'062.700 & 987.550 & 923.940 & 899.425 \\
\hline Población < 75 & $42 ' 995.791$ & $43^{\prime} 488.343$ & 43975629 & $44^{\prime} 460.649$ & $44^{\prime} 953.433$ & $45^{\prime} 309.818$ & $45^{\prime} 810.460$ & $46 ’ 317.378$ & 46 '829.173 \\
\hline APVP por 100.000 & $2.045,5$ & $1.964,6$ & $2.564,7$ & $2.568,7$ & $2.454,1$ & 2.345 & 2.155 & 1.994 & 1.920 \\
\hline
\end{tabular}

APVP: años perdidos de vida potencial 


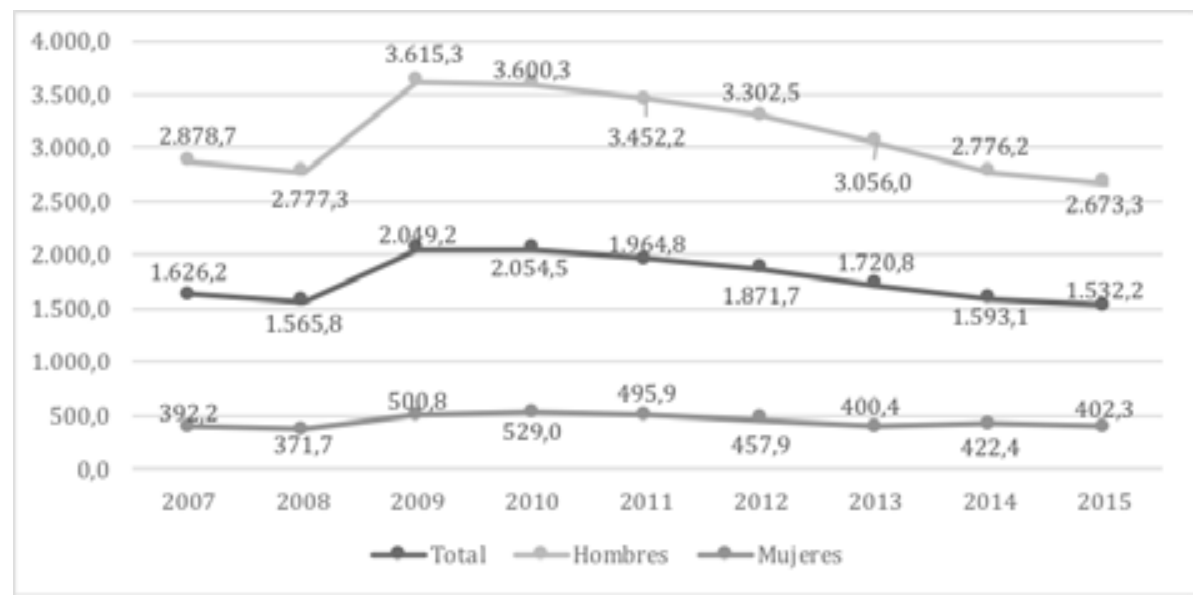

Figura 1. Años perdidos de vida potencial por año, en totales y para cada sexo

\section{Discusión}

Existen pocas experiencias disponibles en la literatura que realicen la descripción del impacto mediante el uso de los "años de vida potencialmente perdidos" (sic). Esta es una medida reconocida y difundida por organizaciones nacionales e internacionales que permiten la evaluación del impacto de muertes relacionadas a eventos no trasmisibles de interés en salud pública ${ }^{15}$.

Esta estimación se basa en los años que una persona no vive según la expectativa de vida de la región en la que se presenta y su interpretación está relacionada a indicadores de inequidades en salud y estado de bienestar basados en datos individuales ${ }^{3-5,12,14,16}$. Con relación a esto, el uso de esta medida de impacto permite identificar el panorama en salud, establecer potenciales estrategias de prevención y establecer las referencias para evaluar el impacto de dichas estrategias ${ }^{17,18}$.

Para realizar el presente estudio se analizaron los datos disponibles por parte de las entidades nacionales oficiales que se encargan de la vigilancia y el seguimiento de las muertes por causa externa y del censo nacional, siendo esta una oportunidad para generar conocimiento por medio del uso de la información disponible y el análisis secundario ${ }^{13}$. Los resultados de nuestro estudio evidencian el compromiso diferencial de sexo, un mayor impacto en las edades econó- micamente productivas y un predominio de los homicidios en la población general.

El comportamiento de las lesiones violentas sobre otros mecanismos de trauma, es un fenómeno que se describe de manera consistente con otras experiencias cuya realidad nacional es similar a la nuestra ${ }^{19,20}$. En Colombia, este fenómeno ha sido reconocido y descrito por otras experiencias basado en datos individuales y experiencias regionales ${ }^{13,14,21}$. En respuesta a este fenómeno social, se han adoptado normas que regulan el porte de armas, el expendio de bebidas embriagantes y medidas de convivencia ciudadana. $\mathrm{La}$ instauración de estas normas puede contribuir a la explicación de la reducción comparativa en las proporciones observadas de muertes relacionadas a los homicidios en los últimos años del periodo analizado ${ }^{22,23}$. La plausibilidad de esta relación ha sido explicada por medio de otros reportes, basados en datos individuales y experiencias regionales, destacando la relación entre la portabilidad de armas y la reducción de los horarios permitidos para el expendio de alcohol, con una disminución de los eventos violentos ${ }^{24,25}$.

Concomitante al impacto en las lesiones violentas, se encuentra la explicación del compromiso de las personas en las edades económicamente productivas, las cuales, a su vez, se relacionan a la etnia y el índice de masculinidad entre otras ${ }^{26,27}$. 
Esta relación se encuentra en consideración a la exposición en el uso de medios de transporte y la necesidad de desplazamiento para las actividades económicas y educativas ${ }^{28,29}$. Basados en esta misma metodología, se ha reconocido la importancia clínica en la reducción de estas lesiones, cuyas características se asocian con mayor morbimortalidad ${ }^{30-32}$.

Las limitaciones de nuestro estudio se encuentran relacionadas con la dinámica del análisis estadístico, en el cual no se tuvieron en cuenta el peso de las categorías extremas y la exposición diferencial según la edad de presentación, y no se propusieron otros ajustes estadísticos para tal fin ${ }^{5}$. Sin embargo, esta es una estimación descrita ampliamente para lesiones relacionadas por trauma y hacen parte de la estimación de la carga de la enfermedad 4,14,19,33.

El impacto de las lesiones por trauma en Colombia, y específicamente en su mortalidad, requiere de estrategias en las que intervengan las entidades territoriales, académicas y de innovación, entre otras; diferentes experiencias han descrito una modificación de estos desenlaces mediante la creación de centros de atención especializados, desarrollo de tecnología, ordenamientos, disposición físicas, normas y disposiciones que permitan el abordaje pertinente de este problema de salud pública ${ }^{34-41}$. Particularmente sobre el desarrollo de tecnología, centros especializados y calidad en la atención, el desarrollo de tecnologías orientadas a la disponibilidad de los servicios quirúrgicos y los sistemas de atención en trauma son factores reconocidos a nivel mundial, cuyo impacto se encuentra reflejado en las tasas globales de complicaciones y mejoría de la supervivencia ${ }^{21,40,42,43}$.

Para concluir, este estudio refleja la realidad nacional frente a la mortalidad de las lesiones por trauma, según las estadísticas oficiales del país. Se destaca de manera consistente en este periodo de nueve años, el predominio del impacto en los hombres jóvenes en edades económicamente productivas y la carga de la enfermedad por lesiones violentas. Las políticas nacionales pueden contribuir a la explicación de la discre- ta reducción de dichos eventos en este periodo, así como la oportunidad para la organización y el desarrollo de servicios especializados para el abordaje de estas lesiones.

\section{Cumplimiento de normas éticas}

Consentimiento informado: este estudio es una revisión de una base de datos estadística, y como tal no hay necesidad de un consentimiento informado por parte de los pacientes ni de aprobación del Comité de Ética Institucional.

Conflictos de interés: ninguno declarado.

Fuentes de financiación: autofinanciado

\section{Referencias}

I. Sarkar B, Brunsvold ME, Cherry-Bukoweic JR, Hemmila MR, Park PK, Raghavendran K, et al. American College of Surgeons' Committee on Trauma Performance Improvement and Patient Safety program: Maximal impact in a mature trauma center. J Trauma. 20II;7I:I447-53. doi:I0.I097/TA.oboI3e3I82325d32.

2. Lustenberger T, Inaba K, Schnüriger B, Barmparas G, Eberle BM, Lam L, et al. Gunshot injuries in the elderly: patterns and outcomes. A national trauma databank analysis. World J Surg. 20II;35:528-34. doi:IO.IOO7/ soo268-0I0-0920-7.

3. Pan American Health Organization. Techniques to measure the impact of mortality: years of potential life lost. Series PAHO. Washington, D.C.: Pan American Health Organization. Epidemiological Bulletin. 2003;24. Fecha de consulta: I2 de julio de 20I8. Disponible en: http:// iris.paho.org/xmlui/handle/123456789/4II99.

4. Rehm J, Patra J, Popova S. Alcohol-attributable mortality and potential years of life lost in Canada 200I: Implications for prevention and policy. Addiction. 2006;IOI:373-84. doi:IO.IIII/j.I360-0443.2005.01338.x.

5. Gardner JW, Sanborn JS. Years of potential life lost (YPLL)--what does it measure? Epidemiology. I990;I:322-9. doi:I0.I097/00ooI648-199007000-00oI2.

6. Holbrook TL, Hoyt DB. The impact of major trauma: Quality-of-life outcomes are worse in women than in men, independent of mechanism and injury severity. J Trauma. 2004;56:284-90. doi:Io.I097/oI. TA.oooolo9758.75406.F8.

7. Al-Koudmani I, Darwish B, Al-Kateb K, Taifour Y. Chest trauma experience over eleven-year period at al-mouassat university teaching hospital-Damascus: A retrospective review of 888 cases. J Cardiothorac Surg. 20I2;7:35. doi:IO.II86/I749-8090-7-35.

8. Mefire AC, Pagbe JJ, Fokou M, Nguimbous JF, Guifo ML, Bahebeck J. Analysis of epidemiology, lesions, 
treatment and outcome of 354 consecutive cases of blunt and penetrating trauma to the chest in an African setting. S Afr J Surg. 2010;48:90-3.

9. Demetriades D, Murray J, Sinz B, Myles D, Chan L, Sathyaragiswaran L, et al. Epidemiology of major trauma and trauma deaths in Los Angeles County. J Am Coll Surg. I998;187:373-83.

Io. Ghosh P, Halder SK, Paira SK, Mukherjee R, Kumar SK, Mukherjee SK. An epidemiological analysis of patients with abdominal trauma in an eastern Indian metropolitan city. J Indian Med Assoc. 20II;IO9:19-23.

II. Murray CJL, Vos T, Lozano R, Naghavi M, Flaxman AD, Michaud C, et al. Disability-adjusted life years (DALY) for 29I diseases and injuries in 2I regions, 1990-2010: A systematic analysis for the Global Burden of Disease Study 20Io. Lancet. 2013;380:2197-223. doi:IO.IOI6/SoI4O6736(I2)6I689-4.

I2. Semerl JS, Sesok J. Years of potential life lost and valued years of potential life lost in assessing premature mortality in Slovenia. Croat Med. J 2002;43:439-45.

I3. Instituto Nacional de Medicina Legal y Ciencias Forenses. Datos para la vida. Forensis. 2015;16:23-5.

I4. Moreno C, Cendales R, Moreno C, Cendales R. Mortality and years of potential life lost due to homicide in Colombia, 1985-2006. Rev Panam Salud Pública. 20II;30:342-53. doi:IO.I590/SIO2O-498920IIOoIOoooo8.

I5. Organización Panamericana de la Salud. Conjunto de datos basicos, expandidos y opcionales para las enfermedades crónicas no transmisibles, violencia y lesiones. Sección I. Causa externa. Washington, D.C.: Organización Panamericana de la Salud; 2009. p. 3748. Disponible en: http://wwwI.paho.org/hq/dmdocuments/2009/violenceinjurydatasetES.pdf.

I6. Kottke TE, Gallagher JM, Rauri S, Tillema JO, Pronk NP, Knudson SM. New summary measures of population health and well-being for implementation by health plans and accountable care organizations. Prev Chronic Dis. 20I6;13:I60224. doi:I0.5888/pcdı3.I60224.

I7. Cerdá M, Morenoff JD, Hansen BB, Tessari Hicks KJ, Duque LF, Restrepo A, et al. Reducing violence by transforming neighborhoods: a natural experiment in Medellín, Colombia. Am J Epidemiol. 2012;175:IO4553. doi:IO.IO93/aje/kwr428.

18. Marchant P. What is the contribution of street lighting to keeping us safe? An investigation into a policy. Radic Stat. 20IO;IO2:32-42.

19. Trunkey DD. Trauma. Sci Am. 1983;249:28-35.

20. Cavazos-Ortega N, Rio-Zolezzi A, Izazola-Licea JA, Lezana-Fernández MA, Valdespino-Gómez JL. Años de vida potencial perdidos: Su utilidad en el análisis de la mortalidad en México. Salud Pública Mex. 1989;31:6Io24.

2I. Ordóñez CA, Pino LF, Tejada JW, Badiel M, Loaiza JH, Mata L V, et al. Experience of two first level hospitals in the southwest region of Colombia on the implementa- tion of the Panamerican Trauma Society International Trauma Registry. Rev Col Bras Cir. 2012;39:255-62.

22. Ministerio de Denfensa Nacional. Decreto 2535 de 1993. Colombia: Suin-Juriscol; 1993. p. 8. Fecha de consulta: I8 de mayo de 20I9. Disponible en: http://www.suin-juriscol.gov.co/viewDocument.asp?id=I46I503.

23. Presidencia de la Republica. Ley Seca. Decreto 3569. Colombia: Departamento Administrativo de Función Pública; 20II. p. I-4. Fecha de consulta: I8 de mayo de 2019. Disponible en: http://www.funcionpublica.gov.co/ eva/gestornormativo/norma.php? $\mathrm{i}=44 \mathrm{I} 29$.

24. Sánchez AI, Villaveces A, Krafty RT, Park T, Weiss HB, Fabio A, et al. Policies for alcohol restriction and their association with interpersonal violence: a time-series analysis of homicides in Cali, Colombia. Int J Epidemiol. 20II;40:I037-46. doi:IO.IO93/ije/dyro5I.

25. El Tiempo. Bajan homicidios en Cali durante la ley zanahoria. 1998. El Tiempo, 30 de agosto de 1998. Fecha de consulta: I7 de noviembre de 2015. Disponible en : http://www.eltiempo.com/archivo/documento/MAM823485

26. Karch DL, Logan J, McDaniel D, Parks S, Patel N. Surveillance for violent deaths--National Violent Death Reporting System, I6 states, 2009. MMWR Surveill Summ. 20I2;6I:I-43.

27. Centers for Disease Control and Prevention. National Center for Injury Prevention and Control. Atlanta, GA: CDC Injury, 2006. Fecha de consulta: I7 de mayo de 2017 Disponible en: https://www.cdc.gov/HomeandRecreationalSafety/Water-Safety/waterinjuries-factsheet.htm

28. Calle-Toro JS, Ordóñez C, Sánchez AI, Sanjuán J, Badiel M, Pino L, et al. Epidemiología de las lesiones relacionadas con colisiones de vehículos motorizados en dos centros de referencia del suroccidente colombiano. Panam J Trauma, Crit Care \& Emerg Surg. 20I4;3:I6-22. doi:I0.5005/jp-journals-I0030-IO80.

29. Comité de Ensambladoras de Motocicletas Japonesas. Séptimo estudio sociodemográfico de los usuarios de motos en Colombia ,2012. Fecha de consulta: 20 de mayo de 20I8. Disponible en: https://www.academia. edu/2I856546/Séptimo_Estudio_Sociodemográfico_de_ los_usuarios_de_motos_en_Colombia_SÉPTIMO_ESTUDIO_SOCIODEMOGRÁFICO_DE_LOS_USUARIOS_DE_MOTOS_EN_COLOMBIA.

30. Botache WF, Ordóñez CA, Badiel M, Sanjuán J, Tejada J, Cepeda MC, et al. Herida por arma de fuego como predictor independiente de mortalidad en trauma de Tórax. Panam J Trauma, Crit Care \& Emerg Surg. 2012;1:198-203.

3I. Murano T, Mohr AM, Lavery RF, Lynch C, Homnick AT, Livingston DH. Civilian craniocerebral gunshot wounds: An update in predicting outcomes. Am Surg. 2005;7I:I009-I4.

32. Lichte P, Oberbeck R, Binnebösel M, Wildenauer R, Pape HC, Kobbe P. A civilian perspective on ballistic 
trauma and gunshot injuries. Scand J Trauma Resusc Emerg Med. 20I0;I8:35. doi:Io.II86/I757-724I-I8-35.

33. Lozano R, Naghavi M, Foreman K, Lim S, Shibuya K, Aboyans $\mathrm{V}$, et al. Global and regional mortality from 235 causes of death for 20 age groups in 1990 and 2010: A systematic analysis for the Global Burden of Disease Study 20Io. Lancet. 2012;380:2095-I28. doi:IO.IOI6/SoI4O6736(12)6I728-0.

34. Peek-Asa C. Role of environmental interventions in injury control and prevention. Epidemiol Rev. 2003;25:7789. doi:Io.Io93/epirev/mxgoo6.

35. Laufer W, Adler F. The criminology of criminal law. First edition. New York: Taylor \& Francis; 2012.

36. Northridge ME, Sclar ED, Biswas P. Sorting out the connections between the built environment and health: A conceptual framework for navigating pathways and planning healthy cities. J Urban Health. 2003;80:556-68. doi:Io.I093/jurban/jtgo64.

37. Loomis D, Marshall SW, Wolf SH, Runyan CW, Butts JD. Effectiveness of safety measures recommended for prevention of workplace homicide. JAMA. 2002;287:IOII-7.

38. Rogers FB, Osler T, Lee JC, Sakorafas L, Wu D, Evans $\mathrm{T}$, et al. In a mature trauma system, there is no differ- ence in outcome (survival) between Level I and Level II trauma centers. J Trauma. 20II;70:I354-7. doi:Io.I097/ TA.oboi3e3182I83789.

39. Lendrum RA, Lockey DJ. Trauma system development. Anaesthesia. 2013;68 (Suppl.I):30-9. doi:Io.IIII/ anae.I2049.

40. Odriozola FA, Berroeta FA. Impact of implementation of a trauma care system in severe trauma mortality. Med Intens. 2006;30:336-8.

4I. Tubb CC, Oh JS, Do NV, Tai NR, Meissel MP, Place ML. Trauma care at a multinational United Kingdom-led Role 3 combat hospital: Resuscitation outcomes from a multidisciplinary approach. Mil Med. 20I4;I79:I258-62. doi:I0.7205/MILMED-D-I4-00042.

42. Bailey J, Trexler S, Murdock A, Hoyt D. Verification and regionalization of trauma systems: The impact of these efforts on trauma care in the United States. Surg Clin North Am. 20I2;92:IOO9-24. doi:IO.IOI6/j. suc.2012.04.008.

43. Gray SF, Leung GM. Investing in Health. J Public Health (Oxf). 2006;34:319. doi:IO.Io93/pubmed/fdso73. 